\title{
Audição e percepção da perda auditiva em idosos
}

\section{Hearing and perception of hearing loss in elderly people}

\author{
Maria da Glória Canto de Sousa ${ }^{1}$, Iêda Chaves Pacheco Russo ${ }^{2}$
}

\begin{abstract}
RESUMO
Objetivo: Avaliar a audição e a percepção dos indivíduos idosos sobre a sua condição de audição. Métodos: Foi desenvolvida uma pesquisa descritiva e exploratória, tendo como sujeitos 40 alunos, sendo 34 do sexo feminino e seis do sexo masculino, com idades variando entre 61 e 88 anos, de uma Universidade Aberta à Terceira Idade, localizada na cidade do Salvador, na Universidade do Estado da Bahia. Para avaliação dos sujeitos foi aplicado o Inventário Auditivo para Idosos - IAPI e realizado o exame audiométrico. A análise dos dados foi feita a partir das variáveis: sexo, faixa etária e pela computação das respostas dadas pelos indivíduos ao Inventário Auditivo para Idosos. Resultados: Dos idosos 37,5\% apresentaram audição normal e 62,5\% perda auditiva, sendo 30\% perda auditiva assimétrica e 32,5\% simétrica. Dos 25 sujeitos com perda auditiva, apenas 3 (12\%) tiveram percepção da mesma com um IAPI superior a dez pontos e 22 (88\%) com uma pontuação inferior a dez. Quanto ao sexo, $8 \%$ de homens e $4 \%$ de mulheres tiveram uma pontuação superior a dez no IAPI; $16 \%$ dos indivíduos do sexo masculino e $72 \%$ do feminino apresentaram pontuação inferior a dez. Houve um predomínio da configuração audiométrica do tipo descendente em $88 \%$ dos sujeitos. Conclusão: A maioria dos sujeitos estudados era portadora de perda auditiva, sendo que, quanto maior a idade, maior esta deficiência. Proporcionalmente, a perda auditiva ocorreu mais em homens do que em mulheres, mas poucos percebem a sua existência.
\end{abstract}

Descritores: Idoso; Envelhecimento; Presbiacusia; Transtornos da audição; perda auditiva; Qualidade de vida

\section{INTRODUÇÃO}

A comunicação se traduz em uma necessidade vital do ser humano. É por meio da comunicação que o indivíduo mantém as possíveis trocas em suas relações sociais, permitindo o aproveitamento pleno das experiências já vividas. Comunicar é compartilhar idéias e pensamentos por meio da linguagem, sendo a linguagem falada a mais utilizada no mundo e, para tanto, entre outros aspectos, é necessária a preservação da audição ${ }^{(1)}$.

Cada vez mais o tema envelhecimento vem sendo abordado, tanto nos países desenvolvidos quanto nos países em desenvolvimento. No Brasil, o envelhecimento da população é um fenômeno relativamente novo e os estudos sobre o tema não são ainda numerosos.

A senescência é algo natural e irreversível e, com o passar dos anos, os indivíduos sofrem mudanças, tanto nos aspectos

Trabalho realizado na Universidade Aberta à Terceira Idade (UATI) da Universidade do Estado da Bahia - UNEB - Salvador (BA), Brasil.

(1) Mestre, Professora do Curso de Fonoaudiologia da Universidade do Estado da Bahia - UNEB - Salvador (BA).

(2) Doutora, Professora do Programa de Estudos Pós-Graduados em Fonoaudiologia da Pontifícia Universidade Católica de São Paulo - PUC-SP - São Paulo (SP), Brasil e do Curso de Fonoaudiologia da Faculdade de Ciências Médicas da Santa Casa de São Paulo - FCMSCSP - São Paulo (SP), Brasil.

Endereço para correspondência:

Maria da Glória Canto de Sousa. R. Ítalo Gaudenzi, 3, Stella Mares, Salvador

- BA, CEP: 41600-470. E-mail: gloriacanto@uol.com.br

Recebido em: 24/03/2008; Aceito em: 22/2/2009 biológico, como social e psicológico. Este processo consiste na deterioração lenta e progressiva das funções orgânicas, que pode ou não ser imprescindível à manutenção da vida.

Estudos comprovam que a perda auditiva na população idosa ocorre de 5 a $20 \%$ nos indivíduos com 60 anos de idade; essa incidência aumenta para $60 \%$ nos indivíduos a partir dos $65 \operatorname{anos}^{(2)}$.

A deficiência auditiva no idoso, denominada de presbiacusia, resulta em efeitos negativos não só do ponto de vista social e emocional, como também na qualidade de vida do idoso $^{(3-5)}$. É um dos distúrbios da comunicação mais incapacitantes, impedindo-lhe de desempenhar o seu pleno papel na sociedade, porque não só provoca uma privação sensorial, mas acarreta uma dificuldade de compreensão da fala daqueles que o cercam, dificultando a comunicação ${ }^{(6)}$.

Dependendo do tipo e grau de comprometimento das estruturas sensoriais da orelha interna, bem como das estruturas neurais $^{(7)}$, a interferência da inteligibilidade da palavra poderá ocorrer em maior ou menor grau.

É comum na população idosa a dificuldade de compreensão da fala, principalmente em situações de comunicação desfavoráveis como em ambientes ruidosos ${ }^{(8-11)}$.

Estudos revelam que existe uma desproporção entre as queixas auditivas do idoso e sua real condição auditiva ${ }^{(12)}$; no entanto, existe um consenso quanto ao processo degenerativo na audição da população idosa ${ }^{(9)}$.

Uma preocupação comum na população idosa do sexo feminino diz respeito ao aparecimento das manifestações au- 
ditivas, quando comparada ao sexo masculino, pois apresenta maior percepção da desvantagem auditiva ${ }^{(9-13)}$.

No século XXI, o maior desafio será o de cuidar de uma população de mais de 32 milhões de idosos que, em sua maioria, apresenta nível sócio-econômico e educacional desfavorável. Desta forma, faz-se necessária a adoção de medidas prioritárias de saúde e bem-estar desta população crescente por meio de ações concentradas de promoção de saúde ${ }^{(14)}$.

Nos Estados Unidos, a presbiacusia é considerada como um verdadeiro problema de saúde pública, já que aproximadamente $30 \%$ dos americanos com mais de 65 anos admitem ter perda auditiva, o que corresponde a nove milhões de pessoas ${ }^{(15-16)}$.

A atuação do fonoaudiólogo junto a idosos vem cada vez mais contribuir para otimizar os aspectos biopsicossociais, principalmente quando esta faz parte de um processo interdisciplinar. Os recursos utilizados para a reabilitação auditiva auxiliam no sentido de minimizar a dificuldade de comunicação, uma vez que possibilitam a melhora no desempenho auditivo, proporcionando maior integração do deficiente auditivo à sociedade.

Desta forma, levar ao conhecimento da população idosa informações que possam contribuir para desencadear uma percepção "precoce" das condições auditivas possibilitará uma intervenção que possa evitar ou minimizar as implicações psicossociais e o isolamento do indivíduo da sociedade.

Diante do exposto, os objetivos desta pesquisa foram avaliar a audição e verificar a percepção que indivíduos idosos, alunos de uma Universidade aberta a Terceira Idade, têm sobre a sua condição auditiva.

\section{MÉTODOS}

A pesquisa foi realizada na Universidade Aberta à Terceira Idade (UATI), da Universidade do Estado da Bahia - UNEB, localizada na cidade do Salvador, no município do Cabula.

Inicialmente, a pesquisadora entrou em contato com a dirigente da UATI, a fim de obter autorização para realizar a pesquisa via carta de solicitação. Todos os participantes foram convidados, por intermédio da dirigente, a participar do estudo. Um termo de consentimento livre e esclarecido foi lido e devidamente assinado pelos sujeitos, anteriormente ao início do estudo. Este projeto foi encaminhado ao Comitê de Ética em Pesquisa do Programa de Estudos Pós-Graduados em Fonoaudiologia da Pontifícia Universidade Católica de São Paulo, tendo sido aprovado sob parecer de no ${ }^{\circ}$ 045/2006.

A população do estudo foi definida por amostragem probabilística, do tipo casual simples, na qual cada um dos 400 sujeitos elegíveis foi escolhido aleatoriamente, tendo assim, a mesma chance de seleção. Ao final, a população foi de 40 sujeitos, o que representou cerca de $10 \%$, dos sujeitos elegíveis, inscritos na instituição estudada. Dos 40 sujeitos avaliados, 34 eram do sexo feminino e seis do sexo masculino, com idades variando entre 61 e 88 anos.

Os instrumentos utilizados na pesquisa constaram de um roteiro de entrevista, elaborado pela pesquisadora, contendo 11 itens e um questionário denominado Inventário Auditivo para Idosos (IAPI) traduzido e adaptado ${ }^{(17)}$ passando a contar com 14 perguntas.
A escolha do roteiro de entrevista deu-se a partir da intenção de verificar as possíveis doenças já diagnosticadas nos idosos, além das atividades de lazer, praticadas por eles, buscando relacionar saúde e lazer com a perda auditiva. A entrevista abordou os seguintes dados: identificação pessoal (cor da pele, grau de escolaridade, estado conjugal, cor da pele, ocupação); prática de atividade física; lazer; problemas de saúde em geral com diagnóstico médico relacionado a problemas cardíacos, diabetes, hipertensão arterial, distúrbio em coluna cervical, distúrbio visual e do sono de ansiedade. Desta forma, pensou-se na relação de algumas dessas variáveis na apresentação dos resultados.

O IAPI foi utilizado com o objetivo de relacioná-lo com os achados audiológicos, em função das variáveis: sexo, idade, grau e simetria da perda auditiva. Para analisar o IAPI, foi empregada uma escala de quatro pontos e as respostas às perguntas foram marcadas com um " $X$ " que correspondeu a: quase sempre (3), metade do tempo (2), ocasionalmente (1) ou nunca (0).

Assim, os idosos que obtivessem uma pontuação superior a 10 pontos teriam percepção da perda auditiva, sendo o inverso, uma pontuação inferior a 10 pontos, a não percepção da perda auditiva.

A avaliação audiométrica incluiu a determinação dos limiares tonais, por via aérea e por via óssea, sendo os audiogramas classificados quanto ao grau de perda auditiva, simetria e assimetria. A classificação do grau da perda auditiva obedeceu a critérios previamente estabelecidos ${ }^{(18)}$. Para a análise dos limiares audiométricos, foram considerados dentro da faixa de normalidade aqueles que se encontravam em até $25 \mathrm{~dB}$ NA (decibel Nível de Audição). No entanto, considerando os critérios estabelecidos ${ }^{(18)}$, os quais preconizam o grau da perda auditiva a partir da média entre as frequências de 500, 1000 e $2000 \mathrm{~Hz}$ e que a perda auditiva em idosos acontece nas frequências mais altas, optamos por usar a média dos limiares obtidos em 3000, 4000, 6000 e $8000 \mathrm{~Hz}$ para a obtenção do grau de perda auditiva ${ }^{(17)}$. Desta forma, foram utilizados os mesmos valores de classificação de perda auditiva ${ }^{(18)}$, porém com variação das frequências.

Estes resultados, por sua vez, foram correlacionados aos resultados obtidos na aplicação do IAPI.

As inferências estatísticas para verificar as diferenças de proporções foram feitas por meio do Teste Exato de Fisher e o pacote estatístico utilizado foi o R 2.0.1. Em todas as análises, foi fixado o nível de significância em 0,05.

\section{RESULTADOS}

A Tabela 1 apresenta o número e a porcentagem de indivíduos idosos, segundo sexo e idade.

A Tabela 2 apresenta o número e a porcentagem de sujeitos avaliados na audiometria tonal liminar, de acordo com o grau de perda auditiva para as orelhas esquerda e direita.

Na Figura 1 pode ser observado o número dos sujeitos com audição normal e com perda auditiva, em função da faixa etária.

Considerando, ainda, o resultado da distribuição do número de sujeitos com audição normal e com perda auditiva, a Figura 
2 mostra esta relação em função do sexo e da faixa etária.

Na Tabela 3 pode-se observar a distribuição do número e da porcentagem dos sujeitos que apresentaram audição normal, perdas auditivas assimétricas e simétricas.

Com relação ao número e a porcentagem de sujeitos em função da soma obtida nas questões do IAPI, a Tabela 4 apresenta o quanto o sujeito percebe ou não sua dificuldade auditiva.

Na Tabela 5, pode ser encontrada a distribuição dos resultados obtidos a partir da aplicação do IAPI, de acordo
Tabela 1. Distribuição dos sujeitos de acordo com parte dos dados de identificação da entrevista dirigida: sexo, idade

\begin{tabular}{lrc}
\hline Variáveis & $\mathrm{N}=40$ & $100 \%$ \\
\hline Sexo & 34 & 85 \\
Feminino & 6 & 15 \\
Masculino & & \\
Idade (em anos) & 24 & 60 \\
De 61 a 69 & 16 & 40 \\
De 70 a 88 &
\end{tabular}

Legenda: $\mathrm{N}$ = número de sujeitos da população de estudo

Tabela 2. Resultados da audiometria tonal de acordo com o grau de perda auditiva para as orelhas esquerda e direita

\begin{tabular}{|c|c|c|c|c|c|c|}
\hline \multirow[t]{3}{*}{ Grau de perda auditiva } & \multicolumn{4}{|c|}{ Orelha } & \multicolumn{2}{|c|}{ Total } \\
\hline & \multicolumn{2}{|c|}{ Esquerda } & \multicolumn{2}{|c|}{ Direita } & \multirow[b]{2}{*}{$\mathrm{N}=80$} & \multirow[b]{2}{*}{$100 \%$} \\
\hline & $\mathrm{N}=40$ & $100 \%$ & $\mathrm{~N}=40$ & $100 \%$ & & \\
\hline Audição normal & 18 & 45 & 18 & 45 & 36 & 45 \\
\hline Perda auditiva de grau leve & 11 & 27,5 & 8 & 20 & 19 & 23,7 \\
\hline Perda auditiva de grau moderado & 11 & 27,5 & 14 & 35 & 25 & 31,3 \\
\hline
\end{tabular}

Legenda: $\mathrm{N}$ = número de orelhas

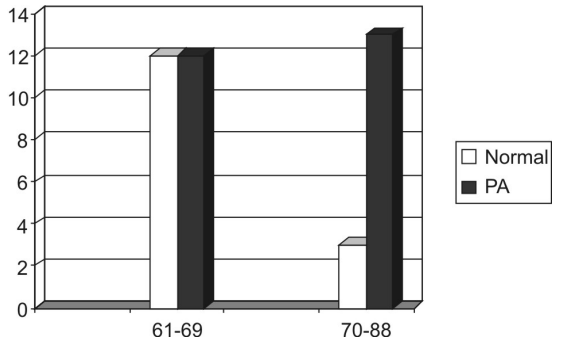

Figura 1. Distribuição dos sujeitos com audição normal (NL) e com perda auditiva (PA) em função da faixa etária

Tabela 3. Número e a porcentagem dos sujeitos que apresentaram audição normal, perdas auditivas assimétricas e simétricas

\begin{tabular}{lcc}
\hline Resultado da audiometria tonal & $\mathrm{N}=40$ & $100 \%$ \\
\hline Audição normal (simétrica) & 15 & 37,5 \\
Perda auditiva (assimétrica) & 12 & 30 \\
Perda auditiva (simétrica) & 13 & 32,5 \\
\hline
\end{tabular}

Legenda: $\mathrm{N}$ = número de sujeitos da população de estudo

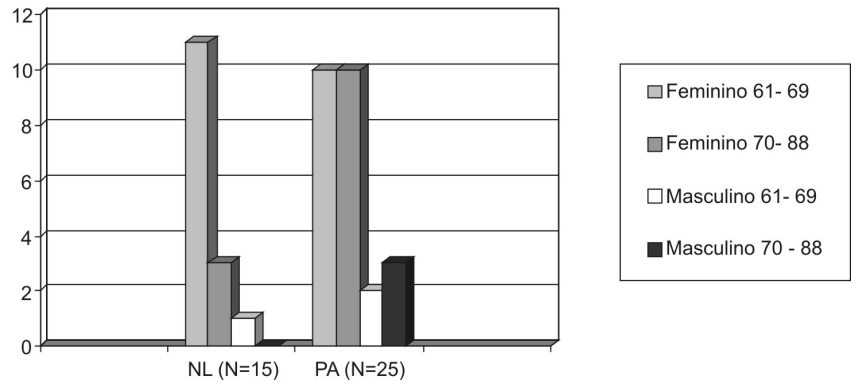

Figura 2. Distribuição dos sujeitos com audição normal (NL) e com perda auditiva (PA) em função do sexo e da faixa etária

Tabela 4. Distribuição dos sujeitos de acordo com a soma das pontuações das questões do IAPI

\begin{tabular}{lcc}
\hline Soma das pontuações do questionário IAPI & $\mathrm{N}=40$ & $100 \%$ \\
\hline De zero a dez & 33 & 82,5 \\
De 11 a 20 & 7 & 17,5 \\
\hline
\end{tabular}

Legenda: $\mathrm{N}$ = número de sujeitos da população de estudo

Tabela 5. Inventário Auditivo para Idosos (IAPI) de acordo com o sexo, a idade e o grau de perda auditiva

\begin{tabular}{|c|c|c|c|c|c|c|c|}
\hline \multirow{3}{*}{ Resultados gerais } & \multicolumn{4}{|c|}{ IAPI } & \multirow{2}{*}{\multicolumn{2}{|c|}{ Total }} & \multirow{3}{*}{ Valor de $p$} \\
\hline & \multicolumn{2}{|c|}{$\leq 10$ pontos } & \multicolumn{2}{|c|}{$>10$ pontos } & & & \\
\hline & $\mathrm{N}=33$ & $\mathrm{P}=82,5$ & $\mathrm{~N}=7$ & $\mathrm{P}=17,5$ & $\mathrm{~N}=40$ & $100 \%$ & \\
\hline \multicolumn{8}{|l|}{ Sexo } \\
\hline Feminino & 29 & 87,9 & 5 & 71,4 & 34 & 85 & 0,2786 \\
\hline Masculino & 4 & 12,1 & 2 & 28,6 & 6 & 15 & \\
\hline \multicolumn{8}{|l|}{ Idade (em anos) } \\
\hline De 61 a 69 & 20 & 60,6 & 3 & 42,9 & 23 & 57,5 & 0,4316 \\
\hline De 70 a 88 & 13 & 39,4 & 4 & 57,1 & 17 & 42,5 & \\
\hline \multicolumn{8}{|l|}{ Audiometria } \\
\hline Audição normal & 12 & 36,4 & 3 & 42,9 & 15 & 37,5 & 0,9999 \\
\hline Perda auditiva de grau leve & 8 & 24,2 & 1 & 14,2 & 9 & 22,5 & \\
\hline Perda auditiva de grau moderado & 13 & 39,4 & 3 & 42,9 & 16 & 40 & \\
\hline
\end{tabular}

Legenda: $\mathrm{N}$ = número de sujeitos da população de estudo; $\mathrm{P}=$ porcentagem dos sujeitos (\%). Observação: Nenhuma diferença foi estatisticamente significante 


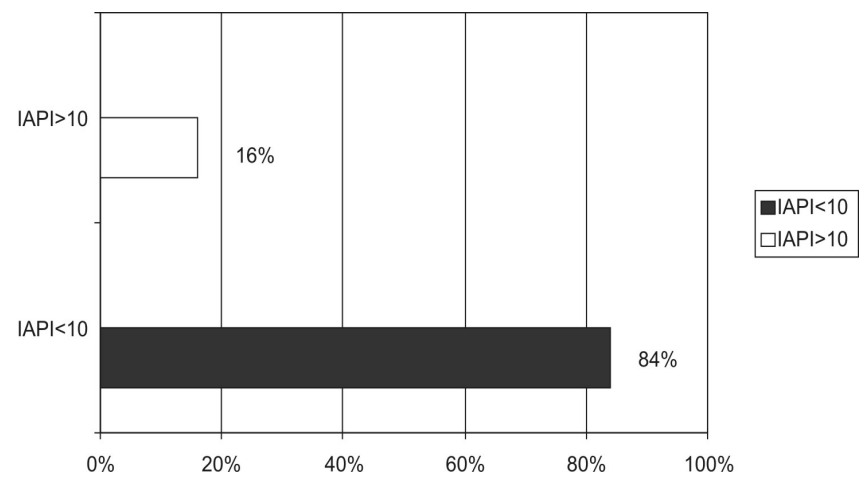

Figura 3. Resultados da pontuação do IAPI para 25 sujeitos que apresentaram perda auditiva

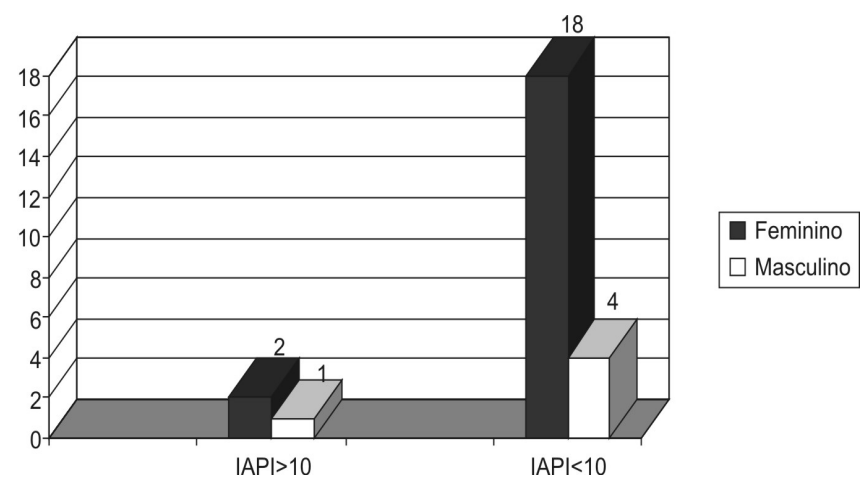

Figura 4. Resultados dos sujeitos com perda auditiva de acordo com o sexo e a pontuação no IAPI

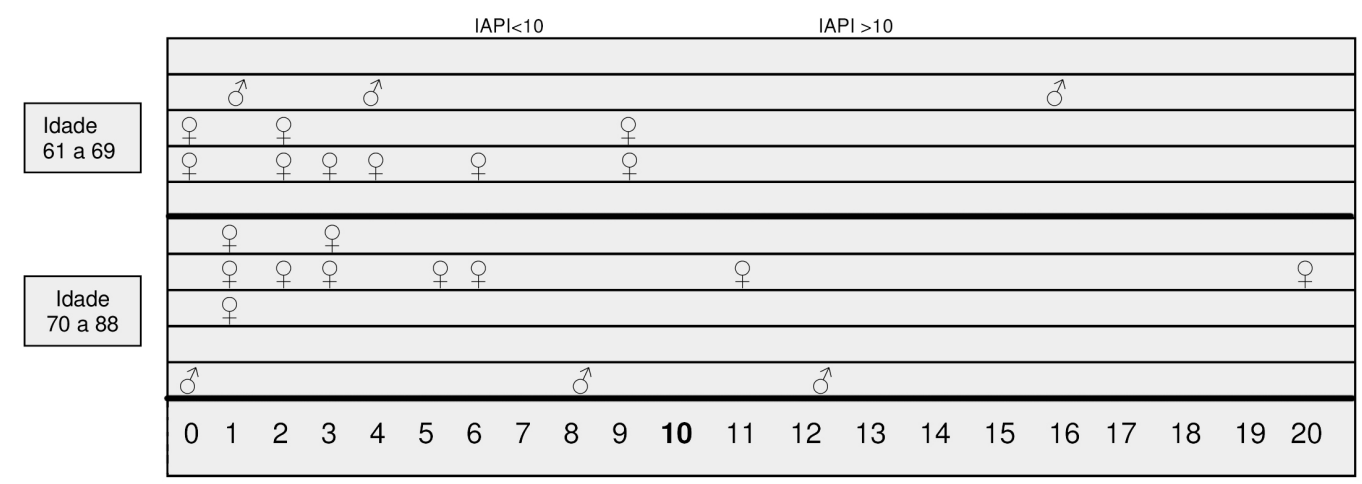

Figura 5. Distribuição de indivíduos com perda auditiva (25) de acordo com a pontuação do IAPI, sexo e idade

com o sexo, a idade e o grau de perda auditiva. No Quadro 1 , encontram-se os níveis descritivos (p-valor) para as inferências estatísticas (Teste Exato de Fisher) das diferenças de proporções.

A Figura 3 ilustra os resultados da pontuação do IAPI para os 25 sujeitos que apresentaram perdas auditivas.

Ainda, considerando a pontuação no IAPI, a Figura 4 mostra os resultados dos sujeitos com perda auditiva de acordo com o sexo.

Finalizando a apresentação dos resultados, a Figura 5 apresenta a distribuição dos 25 sujeitos com perda auditiva, de acordo com a pontuação do IAPI, sexo e idade.

\section{DISCUSSÃO}

O estudo constou de 40 sujeitos, tendo como maioria os do sexo feminino, sendo uma característica inerente aos grupos que se propõem a estudar nas Universidades Abertas à Terceira Idade, conforme demonstrado na Tabela 1 . Na distribuição percentual da amostra, percebe-se que houve grande diferença entre a quantidade de indivíduos do sexo feminino (85\%) e masculino (15\%).

A faixa etária que concentrou maior número de sujeitos foi a de 61 a 69 anos, o que demonstra certa autonomia e independência destes sujeitos por estarem no início da terceira idade (Tabela 1 e Figuras 1 e 2).

Além da avaliação auditiva, que fornece informações acerca da condição auditiva do indivíduo, questões não auditivas podem ser avaliadas por meio de diversos tipos de questionários. Os estudos apontam que, nos últimos anos, indivíduos deficientes auditivos têm se beneficiado da avaliação das dificuldades auditivas e não auditivas por meio de questionários. Estes instrumentos vêm sendo incorporados na rotina clínica, privilegiando a abordagem de aspectos como: questões de detecção precoce dos problemas auditivos; avaliação da auto-percepção do handicap auditivo; coleta de informações acerca da instituição em estudo, dos funcionários e dos idosos, além dos aspectos psicossociais gerados pela deficiência auditiva ${ }^{(17-20)}$.

A descoberta de novas formas de viver e encarar a vida faz renovar o conceito do que seria a velhice. As relações interpessoais estabelecidas entre os indivíduos sofrem influências que refletem na constituição do próprio sujeito, sendo ele passível de absorver conceitos e preconceitos advindos de um mundo, no qual a competência comunicativa é importante na relação entre os interlocutores. Daí a importância de se mudar os paradigmas, pois fazemos parte de um país que está envelhecendo e que novos conceitos e idéias acerca da terceira e quarta idades precisam ser revistos e reformulados ${ }^{(21)}$.

Estudos têm sido feitos e constatado um aumento do número de pessoas idosas ${ }^{(22)}$ e, consequentemente, a maior incidência da presbiacusia ${ }^{(23-24)}$. A dificuldade auditiva não só repercute no ouvir, traz também, no âmbito social, consequências na qualidade de vida do idoso ${ }^{(3-5)}$. Importantes são os esforços na valorização da audição que venham a resultar em melhor qualidade de vida. Acredita-se que o trabalho fonoaudiológico deva ser pautado nas questões sócio-culturais e que as características regionais do país são bastante diversificadas, 
o que nos leva a refletir tais questões, pensando no meio em que o idoso está inserido ${ }^{(21)}$. Daí a necessidade emergente do uso mais eficiente dos recursos públicos direcionados à terceira idade e à problemática da presbiacusia, pois tal fato vem sendo tratado como uma questão de saúde pública no Brasil, com necessidades específicas quanto à promoção de saúde $\mathrm{e}$ reabilitação auditiva para os idosos ${ }^{(8,24)}$.

Em nosso estudo pudemos observar que na Tabela 3, tivemos um índice de 37,5\% de sujeitos com audição normal e $62,5 \%$ de perda auditiva, sendo $30 \%$ perda auditiva assimétrica e 32,5\% simétrica $^{(9)}$. Desta forma, verificamos que este achado vem ratificar a importância de se fazer um trabalho de orientação, no sentido de alertar esta população sobre as possíveis alterações auditivas e de que forma devem ser encaradas e, se possível, prevenidas.

A Figura 2 que descreve o número dos sujeitos com audição normal e com perda auditiva, em função do sexo e da faixa etária, demonstrou que com o aumento da idade, tivemos uma maior ocorrência de perda auditiva no sexo masculino do que no feminino. Portanto, $41,2 \%$ dos sujeitos do sexo feminino apresentavam audição normal, contra 16,6\% do sexo masculino; $83,6 \%$ dos sujeitos do sexo masculino apresentavam perda auditiva, contra 58,8\% do sexo feminino. Estudos revelam maior incidência de normalidade da audição para o sexo feminino e perda neurossensorial para o masculino ${ }^{(25)}$. Ressaltamos, ainda, que o envelhecimento é um fator de risco significativo para a perda auditiva que, com o decorrer da idade, aumenta e que os níveis de audição são levemente piores nos homens do que nas mulheres ${ }^{(26)}$, como demonstrado em nosso estudo.

$\mathrm{Na}$ distribuição percentual dos sujeitos desta pesquisa no que se refere à deficiência auditiva e faixa etária, houve um aumento gradativo da perda auditiva em relação ao aumento da idade ${ }^{(27)}$. Este achado pode ser constatado na Figura 1 o que já foi observado em outro estudo ${ }^{(28)}$. Já em relação ao sexo, houve uma discordância com os achados da referida autora. Tal ocorrência pode ser observada na Figura 2.

Considerando uma possível interferência da perda auditiva na qualidade de vida dos idosos, que tinham conhecimento da existência de sua deficiência auditiva, estudos ressaltam que tal interferência possa estar relacionada à somatização da infelicidade gerada pela perda auditiva neurossensorial, considerada como um fator irreversível. Analisando por esse prisma, concordamos com a autora que, ao considerar a presbiacusia como sendo uma perda auditiva irreversível, assumir ser portador desta pode representar grande sofrimento emocional ao idoso.

Na Figura 3, com relação à soma das questões do IAPI e considerando o número total de sujeitos, 33 (82,5\%) apresentaram um IAPI menor que dez pontos e apenas $7(17,5 \%)$ uma pontuação superior a dez. Em outro estudo ${ }^{(20)}, 89 \%$ dos sujeitos percebiam algum tipo de handicap e esta percepção não variou em função do sexo, faixa etária e grau da perda auditiva. Este dado se contrapõe aos nossos achados ao considerarmos o sexo. Verifica-se, no entanto, que considerando os 25 idosos (total) com perda auditiva, tem-se $8 \%$ de homens e $4 \%$ de mulheres com IAPI $>10$ e $72 \%$ das mulheres e $16 \%$ dos homens $<10$, o que ratifica tal discordância da autora, conforme achados de nossa pesquisa descritos na Tabela 5 e Figuras 4 e 5.

No tocante à existência de variabilidade de respostas em relação à autopercepção de idosos com a mesma sensibilidade auditiva, porém com audição normal e com perda auditiva, pudemos observar que, dos seis idosos do sexo masculino, cinco apresentaram perda auditiva; dentre eles, apenas um teve uma pontuação maior que 10 no IAPI. Com relação aos 19 sujeitos do gênero feminino, com perda auditiva, 17 obtiveram pontuação menor que 10 pontos no IAPI e apenas 2 tiveram pontuação superior a 10 pontos no IAPI, conforme a Figura 5. Há uma tendência dos idosos do gênero masculino apresentarem perda auditiva mais acentuada ${ }^{(29-30)} \mathrm{e}$ maior percepção do handicap auditivo do que os do gênero feminino. Este dado corrobora os achados verificados em nossa pesquisa, vez que $83,6 \%$ dos idosos do sexo masculino apresentaram perda auditiva, considerando os dois grupos de faixa etária, assim demonstrado na Figura 2.

No Quadro 1 estão descritos os resultados do IAPI relacionados ao sexo, idade e audiometria. Constatamos que não existe uma relação de dependência entre os mesmos, ou seja, não houve significância estatística. Entretanto, ao discutirmos os resultados, não encontramos na literatura compulsada autores que fizessem referência ao conjunto dessas relações, o que nos induz à reflexão sobre a influência da audição na qualidade de vida do idoso. Necessitamos estar em sintonia com essa população, a fim de contribuir com nossa parcela de conhecimento e ajudar os que estão hoje no nosso lugar amanhã.

\section{CONCLUSÃO}

A maioria dos sujeitos estudados era portador de perda auditiva, sendo que quanto maior a idade, maior o grau. Proporcionalmente, a perda auditiva ocorreu mais para os indivíduos do sexo masculino do que para o feminino. Apesar da maioria dos sujeitos apresentarem perda auditiva, poucos têm a percepção da mesma. 


\begin{abstract}
Purpose: To evaluate the hearing of elderly individuals and their perception regarding their hearing condition. Methods: A descriptive and exploratory research was developed using as subjects 40 students from a University for the Third Age located in Salvador, Bahia (Brazil), being 34 female and 6 male, with ages ranging from 61 to 88 years. The evaluation used the Hearing Handicap Inventory for Elderly - HHIE, and an audiometric assessment was carried out. Data analysis considered the following variables: gender, age and the answers computed in the HHIE. Results: From the subjects, 37.5\% had normal hearing and 62.5\% presented hearing loss (30\% asymmetric and $32.5 \%$ symmetric hearing loss). From the 25 subjects with hearing loss, only $3(12 \%)$ were aware of it, according to the HHIE, scoring higher than ten points. In addition, 22 subjects $(88 \%)$ had a score lower than ten. Regarding gender, $8 \%$ of the male subjects and $4 \%$ of the female subjects scored over ten at the HHIE; $16 \%$ of the male and $72 \%$ of the female scored under ten points. There was a predominance of the descendent audiometric configuration in $88 \%$ of the individuals. Conclusions: The majority of the subjects studied had hearing loss, which increased with age. Proportionally, hearing loss occurred mostly in men, although few of them were aware of its existence.
\end{abstract}

Keywords: Aged; Aging; Presbycusis; Hearing disorders; Hearing loss; Quality of life

\title{
REFERÊNCIAS
}

1. Bertachini L, Gonçalves MJ. Comunicação na terceira idade. Mundo Saúde (1995). 2002;26(4):483-9.

2. Ruschel CV, Carvalho CR, Guarinello AC. A eficiência de um programa de reabilitação audiológica em idosos com presbiacusia e seus familiares. Rev Soc Bras de Fonoaudiol. 2007;12(2):95-8.

3. Macedo LS, Pupo AC, Baleiro CR. Aplicabilidade dos questionários de auto-avaliação em adultos e idosos com deficiência auditiva. Distúrb Comun. 2006;18(1):19-25.

4. Gonçalves CHO, Mota PHM. Saúde auditiva para a terceira idade: comentários sobre um programa de atenção à saúde auditiva. Distúrb Comun. 2002;13(2):335-49.

5. Betlejewski S. [Age connected hearing disorders (presbyacusis) as a social problem]. Otolaryngol Pol. 2006;60(6):883-6. Polish.

6. Russo ICP. Intervenção audiológica no idoso. In: Ferreira LP, Befi-Lopes DM, Limongi SCO, organizadores. Tratado de fonoaudiologia. São Paulo: Roca; 2004. p. 585-96.

7. Tyberghein J. Presbycusis and phonemic regression. Acta Otorhinolaryngol Belg. 1996;50(2):85-90.

8. Viúde A. Fatores associados à presbiacusia em idosos. [Tese]. São Paulo: Faculdade de Saúde Pública da Universidade de São Paulo; 2002.

9. Espmark AK, Rosenhall U, Erlandsson S, Steen B. The two faces of presbyacusis: hearing impairment and psychosocial consequences. Int $\mathrm{J}$ Audiol. 2002;41(2):125-35.

10. Signorini TB, Azevedo MF, Ebel SJ. Perfil audiológico e caracterização das principais queixas de homens e mulheres entre 65 e 95 anos de idade. Distúrb Comun. 1993;5(2):191-208.

11. Pichora-Fuller MK. Cognitive aging and auditory information processing. Int J Audiol. 2003;42 Suppl 2:2S26-32.

12. Bacha SMC, Giglio VP, Ribeiro JML, Souza MV. Perfil fonoaudiológico do idoso institucionalizado. Pró-Fono. 1999;11(2):1-7.

13. Corrêa GF, Russo IPC. Autopercepção do handicap em deficientes auditivos adultos e idosos. Rev CEFAC. 1999;1(1):54-63.

14. Ramos LR. Fatores determinantes do envelhecimento saudável em idosos residentes em centro urbano: Projeto Epidoso, São Paulo. Cad Saúde Pública = Rep Public Health. 2003;19(3):793-8.

15. Cruz Filho NA, Breuel MLF, Campilongo M. Presbiacusia. In: Campos CAH, Costa HOO, editores. Tratado de otorrinolaringologia. São Paulo: Roca; 2003. p. 186-92. v.2
16. Huang T. Age-related hearing loss. Minn Med. 2007;90(10):48-50.

17. Mesquita CDS. Análise da efetividade de um inventário auditivo para idosos [dissertação]. São Paulo: Pontifícia Universidade Católica de São Paulo; 2001.

18. Lloyd LL, Kaplan H. Audiometric interpretation: a manual of basic audiometry. Baltimore: University Park Press; 1978. p.12-7, 217-9.

19. Silveira KMM. A percepção da deficiência auditiva em um grupo de idosos institucionalizados da cidade de Franca-SP. [Dissertação]. São Paulo: Pontifícia Universidade Católica de São Paulo; 1997.

20. Wieselberg MB. A auto-avaliação do handicap em idosos portadores de deficiência auditiva: o uso do H.H.I.E. [Dissertação]. São Paulo: Pontifícia Universidade Católica de São Paulo; 1997.

21. Mac-Kay APMG. Linguagem e envelhecimento. In: Marchesan IQ, Zorzi JL, Gomes ICD, organizadores. Tópicos em fonoaudiologia 1997/1998. São Paulo: Lovise; 1998. p. 415-20.

22. Kalache A, Veras RP, Ramos LR. O envelhecimento da população mundial: um desafio novo. Rev Saúde Pública. 1987;21(3):200-10.

23. Rosenhall U. [Presbyacusis - hearing loss in old age]. Lakartidningen. 2001;98(23):2802-6. Swedish.

24. Veras RP, Mattos LC. Audiologia do envelhecimento: revisão da literatura e perspectivas atuais. Rev Bras Otorrinolaringol. 2007;73(1):128-34.

25. Jurca APK, Pinheiro FCC, Martins KC, Herrera LF, Colleone LM, Saes SO. Estudo do perfil audiológico de pacientes com idade acima de 60 anos. Salusvita. 2002;21(1):51-65.

26. Soncini F, Costa MJ, Oliveira TMT. Perfil audiológico de indivíduos na faixa etária entre 50 e 60 anos. Fono Atual. 2004;7(28):21-9.

27. Calais LL, Borges ACLC, Baraldi GS, Almeida LC. Queixas e preocupações otológicas e as dificuldades de comunicação de indivíduos idosos. Rev Soc Bras Fonoaudiol. 2008;13(1):12-9.

28. Bilton TL. A deficiência auditiva do idoso e sua implicação na comunicação [dissertação]. São Paulo: Pontifícia Universidade Católica de São Paulo; 1989.

29. Pinzan-Faria VM, Iorio MCM. Sensibilidade auditiva e autopercepção do handicap: um estudo em idosos. Distúrb Comun. 2004;16(3):289-99.

30. Baraldi GS, Almeida LC, Borges ACC. Evolução da perda auditiva no decorrer do envelhecimento. Rev Bras Otorrinolaringol. 2007;73(1):6470 . 